\title{
Diarrea aguda con deshidratación de evolución tórpida
}

\author{
DRES.; CERON, A. "; GALlo, A. *; IBANEZ, S. *; MORAlES, R. *; OTTO, M. A. *; PUENTES, R. *; \\ TALESNIK, E. *; y VEJAR, L. *.
}

La diarrea aguda constituye en Chile la tercera causa de muerte en el menor de un año, con una cifra de alrededor de 3.000 fallecidos anuales (1). Los progresos alcanzados son, no obstante evidentes (2), como se comprueba a vía de ejemplo con el descenso de las tasas de 14,8 a 10,7 por mil nacidos vivos entre 1966-72, que representa un ahorro anual de 1.068 vidas (3). La utilización de los servicios de salud que esta afección implica, se refleja en que de 174 hospitalizaciones de niños, de 1-11 meses de edad por cada 1.000 nacidos en Chile 1968, 78 correspondería a diarreas, lo que constitnye una de las tasas más altas del mundo (4).

En el área Sur Oriente de Santiago las diarreas constituyen la segunda causa de consulta médica de lactantes, con una tasa de 0,74 consultas/habitante/año, y la primera causa de hospitalización en este grupo etario, con una tasa de 67,1 por mil habitantes (5). La mayoría de los niños que ingresan a los servicios pediátricos con este diagnóstico tiesen una evolución favorable y están en condiciones de regresar a sus hogares en el plazo de 5-7 días. Pero en algunos los síntomas se prolongan por un tiempo mayor, configurando lo que ha sido designado entre nosotros como evolución tórpida (6).

El objetivo de este trabajo es analizar la incidencia, causa y pronóstico de este cuadro.

\section{MATERIAL Y METODO}

Se revisó las fichas clínicas de todos los niños ingresados a la Unidad de lactantes del Hospital Sótero del Río entre el 1\% de octubre de 1974 y el 28 de febrero de 1975, separándose aquellos en que la causa de hospitalización había sido una diarrea aguda con deshi-

\footnotetext{
* Servicio y Departamento de Pediatría, Hospital de Niños Tosefina Martínez de Ferrari. Servicio Nacional de Salud. Universidad Católica de Chile.
}

dratación. Se excluyeron los niños con diarreas crónicas y con diarrea aguda sin deshidratación, así como aquellos con diarreas con deshidratación, pero secundaria a otra patología de mayor gravedad (meningitis, bronconeumonía, etc.).

Se clasificó a los lactantes según edad y estado nutritivo, aplicando para ello las tablas distribuidas por el grupo normativo pediátrico del SNS, así como los valores de natremia y kalemia en el momento del ingreso.

Todos los lactantes fueron tratados inicialmente con hidratación parenteral según normas nacionales (4) y realimentación posterior con leche Nido al $2 / 3+5 \%$ azúcar $+3 \%$ Nessucar o bien con una mezcla pobre en lactosa, constituida por zanahoria rallada, quesillo, aceite y glucosa, en dosis progresivas. Salvo que las deposiciones fueran francamente disenteriformes, no se indicó antibióticos al ingreso, sino postcriormente, cuando ello parecía neccsario.

Para evaluar la evolución intrahospitalaria, se consideró como normal la hidratación clínica satisfactoria en un plazo de 48 horas y la ausencia de deposiciones líquidas después de las 72 horas del ingreso, clasificando como evolución tórpida a los niños que no cumplieron ambos requisitos.

El criterio seguido para analizar las cau. sas de evolución tórpida fue el siguiente:

a) Infección urinaria: Se consideró confirmada con un urocultivo positivo por punción vesical con un recuento bacteriano de 100.000 o más, col $/ \mathrm{ml}$ en orina obtenida por recolector $\mathrm{y} / \mathrm{o}$ un sedimento con más de 20 piocitos por campo. Si el examen bacteriológico era negativo y el sedimento dudoso, o sea, con $10-20$ piocitos, se anotó como infección urinario sospechada. 
b) Infección intestinal por bacterias enteropatógenas: Se consideró confirmada sólo por un coprocultivo positivo, y fue sospechada si había deposiciones de tipo enterocólico o si se observó una respuesta evidente al tratamiento con antibióticos.

c) Intolerancia secundaria a lactosa: Se sospechó cuando se produjo una normalización inmediata de las deposiciones al suspender lactosa (crema de zanahoria). Se consideró confirmada por un $\mathrm{pH}$ de deposiciones de 5 o menos y/o reacción de Fehling positiva.

d) Septicemia: Se consideró confirmada por 1 mielocultivo positivo y/o 2 hemocultivo positivos o por el hemocultivo con cuadro clínico muy típico y/o por necropsia. Se catalogó como sospechada cuando el cuadro clínico y la respuesta al tratamiento fueron característicos, pero sin comprobación bacteriológica.

\section{RESULTADOS}

El total de ingresos a la Unidad de lactantes en el período de 5 meses, analizados fue de 802, de los cuales en $255(31,8 \%)$ el motivo de ingreso fue diarrea aguda con deshidratación. 21 de estos ingresos correspondieron a segundas o terceras hospitalizaciones de los mismos lactantes en el mismo período.

En tabla I se presenta la distribución por edades de los lactantes hospitalizados, observándose que alrededor del $70 \%$ son menores de 6 meses.

De los 255 niños analizados, $27(10,6 \%)$ ingresaron con signos clínicos de shock, 93 $(36,0 \%)$ con una acidosis clínica, confirmada por laboratorio en varios y $55(21,6 \%)$ con antecedentes o presentación de deposiciones enterocólicas.

Los valores de natremia se han agrupado en la Tabla II, en que se observa que sólo el $60 \%$ son normales, y que hay un $4,7 \%$ ron hipernatremia y un $16,9 \%$ con hiponatremia, en 3 de ellos muy acentuada.

El tipo de evolución intrahospitalaria se aprecia en la Tabla III, con sólo $58 \%$ de evolución considerada normal y un 5,5\% de letalidad.

De los 148 lactantes con evolución normal de su diarrea, 40 estuvieron hospitalizados más de 10 días, que se estableció como límite máximo aceptable. Las razones para ello se muestran en la Tabla IV.
TABLA I

\section{DISTRIBUCION POR EDAD DE LACTANTES CON DIARREA AGUDA CON DESHIDRATACION}

Hospital Sótero del Río, 1974-1975

\begin{tabular}{ccc}
\hline Edad & No casos & Porcentaje \\
\hline-28 ds. & 15 & 5,9 \\
$1-2$ ms. & 45 & 17,6 \\
$3-5$ ms. & 117 & 45,9 \\
$6-8 \mathrm{~ms}$. & 51 & 20,0 \\
$9-11 \mathrm{~ms}$. & 19 & 7,5 \\
$12+\mathrm{ms}$. & 8 & 3,1 \\
\hline TOTAL & 255 & $100 \%$ \\
\hline
\end{tabular}

TABLA II

\section{VALORES DE NATREMIA EN LACTANTES CON DIARREA AGUDA}

Hospital Sótero del Río, 1974-1975

\begin{tabular}{lcc}
\hline $\begin{array}{l}\text { Natremia } \\
\text { (mEq/Lt) }\end{array}$ & No casos & Porcentaje \\
\hline Sin datos & 47 & 18,4 \\
-120 & 3 & 1,2 \\
$120-129$ & 40 & 15,7 \\
$130-149$ & 153 & 60,0 \\
$150 \mathrm{y}+$ & 12 & 4,7 \\
\hline TOTAL & 255 & $100 \%$ \\
\hline
\end{tabular}

TABLA III

\section{EVOLUCION INTRAHOSPITALARIA DE LACTAN- TES CON DIARREA AGUDA}

Hospital Sótero del Río, 1974-1975

\begin{tabular}{lcc}
\hline Tipo evolución & No casos & Porcentaje \\
\hline Normal & 148 & 58,0 \\
Tórpida & 93 & 36,5 \\
Fallecidos & 14 & 5,5 \\
\hline TOTAL & 255 & $100 \%$
\end{tabular}

Se analizó la correlación del tipo de evolución intrahospitalaria con el estado nutritivo y la presencia de signos de shock, acidosis o enterocolitis al ingreso. En la Tabla V se observa que la única diferencia clara de evo- 
TABLA IV

CAUSAS DE PROLONGACION DE hOSPITALIZACION DE LACTANTES CON DIARREA AGUDA DE EVOLUCION NORMAL

Hospital Sótero del Río, 1974-1975

\begin{tabular}{lc}
\hline CAUSAS & N: casos \\
\hline Infecciones (BRN, septicemias, I.U., otitis & \\
enterocolitis, etc.) & 30 \\
Desnutrición severa & 4 \\
Persistencia dep. alteradas & 2 \\
Administrativas & 5 \\
No precisadas & 3 \\
\hline TOTAL & $44^{\circ}$ \\
\hline
\end{tabular}

- Hubo niños con más de una causa, lo que explica discrepancia. lución según el estado nutritivo fue la mayor letalidad en los desnutridos grado III. Es evidente también que los desnutridos avanzados (Grados II y III) suman 152 , o un $60 \%$ del total de pacientes, y los eutróficos son sólo 42 niños, o un 16,5\%.

En la Tabla VI se observa que la presencia de signos de acidosis al ingreso no introduce cambios importantes en la distribución general de los tipos de evolución. En cambio, la existencia de deposiciones enterocólicas se relaciona con un aumento importante de los casos con evolución tórpida y la presencia de shock aumenta en 5 veces el riesgo de morir.

Finalmente, el análisis de las posibles causas de evolución tórpida revela que sólo en 9 casos $(9,7 \%)$ no fue posible precisarla. En los otros niños se pesquisó o sospechó clínicamente 6 grupos de causas, que fueron la infección bacteriana enteropatógena

TABLA V

TIPOS DE EVOLUCION INTRAHOSPITALARIA DE LACTANTES CON DIARREA AGUDA, SEGUN ESTADO NUTRITIVO

Hospital Sótero del Río, 1974-1975

\begin{tabular}{lccccccccc}
\hline $\begin{array}{c}\text { Estado } \\
\text { Nutritivo }\end{array}$ & & \multicolumn{2}{c}{ TIPO } & \multicolumn{3}{c}{ EVOLUCION } \\
& Normal & & & Tórpida & & Fallecidos & \multicolumn{2}{c}{ TOTAL } \\
\hline Eutrofia & No & $\%$ & No & $\%$ & No & $\%$ & No & $\%$ \\
Desn. I & 25 & 59,5 & 15 & 35,7 & 2 & 4,8 & 42 & 100 \\
Desn. II & 33 & 55,0 & 25 & 41,7 & 2 & 3,3 & 60 & 100 \\
Desn. III & 59 & 58,6 & 37 & 37,4 & 4 & 4,0 & 99 & 100 \\
\hline TOTAL & 31 & 58,7 & 16 & 30,2 & 6 & 11,3 & 53 & 100 \\
\hline
\end{tabular}

- Se cxcluye un RN hipoalimentado.

TABLA VI

TIPO DE EVOLUCION INTRAHOSPITALARIA DE LACTANTES CON DIARREA AGUDA, SEGUN EXISTENCLA DE SHOCK, ACIDOSIS Y ENTEROCOLITIS

Hospital Sótero del Río, 1974-1975

\begin{tabular}{|c|c|c|c|c|c|c|c|c|}
\hline \multirow{2}{*}{$\begin{array}{l}\text { Tipo de } \\
\text { evolución }\end{array}$} & \multicolumn{2}{|c|}{ Con shock } & \multicolumn{2}{|c|}{ c/acidosis } & \multicolumn{2}{|c|}{ c/enterocolit. } & \multicolumn{2}{|c|}{ Grupo Total } \\
\hline & No & $\%$ & No & $\%$ & No & $\%$ & No & $\%$ \\
\hline Normal & 9 & 33,3 & 53 & 57,0 & 24 & 43,6 & 145 & 58,0 \\
\hline Tórpida & 11 & 40,7 & 31 & 33,3 & 28 & 50,9 & 93 & 36,5 \\
\hline Fallecidos & 7 & 25,9 & 9 & 9,7 & 3 & 5,5 & 14 & 5,5 \\
\hline TOTAL & 27 & 100 & 93 & 100 & 55 & 100 & 255 & 100 \\
\hline
\end{tabular}


TABLA VII

CAUSAS DE EVOLUCION TORPIDA DE DIARREA AGUDA EN LACTANTES

Hospital Sótero del Río, 1974-1975

\begin{tabular}{lcc}
\hline CAUSA & No casos & Porcentaje \\
\hline $\begin{array}{l}\text { Infección bacteriana E-P } \\
\quad \text { (sospechada) }\end{array}$ & 41 & 44,9 \\
$\begin{array}{l}\text { Infección urinaria } \\
\quad \text { (sospechada) }\end{array}$ & 8 & 15,1 \\
$\begin{array}{l}\text { Infección urinaria } \\
\quad \text { (confirmada) }\end{array}$ & 6 & \\
$\begin{array}{l}\text { Septicemia sospechada } \\
\text { Septicemia confirmada }\end{array}$ & 7 & 16,1 \\
$\begin{array}{l}\text { Intol. sec. a lactosa } \\
\text { sospechada }\end{array}$ & 3 & \\
Intol. sec. a lactosa & & \\
$\quad$ confirmada & 6 & 9,7 \\
Otras infecciones (BRN, & 12 & 12,9 \\
$\quad$ meningitis, otitis, etc.) & 12 & 11,8 \\
Recaídas intrahospitalarias & 11 & 9,7 \\
No precisadas & 9 & \\
\hline TOTAL & $111^{*}$ & \\
\hline
\end{tabular}

- Corresponde a 93 lactantes. Discrepancia por 2 ó más cautsas simultáncas en algunos.

$(44,9 \%)$, infección urinaria $(15,1 \%)$, septicemia $(16,1 \%)$, intolerancia secundaria a lactosa $(9,7 \%)$, otras infecciones y causas $(12,9 \%)$ y recaídas intrahospitalarias.$(11,8 \%)$.

\section{COMENTARIO}

Letalidad: La letalidad de 5,5\% observada en nuestro material parece estar en límites accptables. La revisión de la literatura nacional demuestra que la letalidad alrededor de 1920 era altísima, entre 85-90\%. La introducción de leches industrializadas e hidratación parenteral bajan estas tasas a $60-$ $75 \%$, y la mejoría general del manejo las llevó a valores de $9,6 \%$ en dispepsias y $46,8 \%$ en toxicosis (8) entre $1943-48$ y de $6.10 \%$ alrededor de 1955 ( 7 ). En 1962, Meneghello y col. (9) dan una tasa de $10,2 \%$, que baja a 5,4 en 1966-67 y luego se mantiene entre 2,0 y 2,5 entre 1967-71 (2). Jarpa y cols. (10) dan también una tasa de letalidad de $5,5 \%$ cn 1965-67. La comparación de estas cifras con las de nuestro hospital exige conocer previamente el criterio de selección del material, y en especial si en esos estudios se incluyeron lactantes con otra patología grave (septicemia, bronconeumonías, etc.) agregada al cuadro diarreico.
Factores que influencian la letalidad: Hubo 2 factores que aparecieron influensiando la letalidad en nuestro análisis, el estado de shock al ingreso y la existencia de una desnutrición de grado 3 . El lactante que ingresó con signos de shock hipovolémico tuvo un riesgo de morir 5 veces mayor que el grupo general. Es obvia la importancia de reconocer este cuadro precozmente y tener normas muy definidas para un manejo, que deberá efectuarse en la unidad de cuidados intensivos.

Los niños con desnutrición grado III mostraron también una letalidad con una tendencia claramente mayor que el resto del grupo, aunque sin alcanzar significación estadística. La desnutrición avanzada significó sobre todo un riesgo más alto de enfermar. En tanto que la prevalecencia de lactantes desnutridos grados II y III en el Area Sur Oriente de Santiago no sobrepasa el 5\%, ellos constituyeron el $60 \%$ de las hospitalizaciones por diarreas aguda con deshidratación. Ello significa para este grupo un liesgo 12 veces mayor que el de la población total de lactantes, de ser hospitalizados con esta patología. Diversas publicaciones nacionales v extranjeras coinciden con esta experiencia y estudian diferentes explicaciones para esta relación $(2,7,8,9,10,11,12,13)$.

Evolución tórpida: El criterio que hemos seguido para definir este tipo de evolución coincide con el de Macaya y col. (6) y nos parece ser el que mejor refleja la realidad de nuestros servicios pediátricos. El concepto de diarrea "intratable" de autores norteamericanos (14) corresponde más bien a diarreas crónicas, debidas a causas no determinadas o raras de encontrar entre nosotros.

La proporción de $36 \%$ de nuestros enfermos en este tipo de evolución compara desfavorablemente con el $11 \%$ de Soriano y cols. (15), que es la única referencia bibliográfica que hemos encontrado.

Las causas encontradas como probables o confirmadas para este tipo de evolución, pucden agruparse en la siguienta forma:

a) Infecciones intestinales bacterianas en $\pm 45 \%$.

b) Infecciones extraintestinales (inf. urinarias, septicemias, etc.) en $t+44 \%$ de los casos.

c) Recaídas intrahospitalarias en $\pm 12 \%$ de los casos.

d) Intolerancia a lactosa en $\pm 10 \%$. 
Con frecuencia se encontraron simultáneamente 2 y a veces 3 de estas causas en el mismo nin̄o. En todo caso es evidente que la gran causante de este tipo de evolución fue la infección, ya sea intestinal o extra-intestinal, que se encontró en más del 90\% de Ios enfermos.

Infección intestinal por bacterias enteropatógenas: Las numerosas publicaciones existentes sobre hallazgos bacteriológicos en deposiciones de lactantes con diarrea aguda dan cifras eminentemente variables $(16,17$, $18,19,20,21,22,24)$. En general, se han encontrado gérmenes enteropatógenos en un 20-30\% de los casos y raramente en más de $40 \%$. Pareciera que con la técnica de innunofluorecencia el rendimicnto pucde aumentar considerablemente (23). En nuestro servicio no contamos con bacteriología adecuada, por lo cual nos hemos guiado por el aspecto disenteriforme de las deposiciones y por la respuesta clínica al tratamiento antibiótico. Con este sistema, catalogamos como enterocolitis al $21,5 \%$ de los casos en el momento del ingreso. Estos casos hicicron una evolución tórpida con una frecuencia significativa mayor que el grupo total. En coincidencia con ésto, en el $45 \%$ de los lactantes con cvolución tórpida, se planteó como causa la infección bacteriana enteral.

Dado el mal pronóstico evolutivo de la infección bacteriana intestinal, podría parecer una buena política iniciar tratamiento antibiótico al ingreso en todos los lactantes hospitalizados por diarrea aguda. Esta politica se justificaría además por la dificultad en establecer un diagnóstico etiológico tanto desde el punto de vista clínico como de laboratorio. Las Shigellas y el Coli son muy sen. sibles al ambiente y los cultivos deben practicarse en forma inmediata, al lado de la cama del enfermo, con deposición fresca, tórula rectal o muestra obtenida por rectosigmoisdoscopio (25). Aún así hay un importante número de falsos negativos, al igual que en las Salmonellosis. Respecto a E. Coli, que es la etiología más frecuente, existe actualmente dificultad en establecer su rol patógeno. Se ha establecido que la E. Coli puede actuar: 1) invadiendo la mucosa intestinal, como lo hacen las Shigellas; 2) atravesándola hasta llegar a la lámina propia, como sucede en las Salmonellas (28); y 3 ) produciendo una exotoxina que no produce lesión histopatológica de la mucosa, pero sin alteración enzimática, con eliminación de gran cantidad de líquidos y electrolitos hacia el lumen $(25,26,27,29)$.

Para nuestros propósitos, el mayor inte- rés de estos estudios es que la capacidad de las distintas cepas Coli para producir exotoxina o invadir mucosa no corresponde siempre a las cepas consideradas clásicamente como enteropatógenos. Además las cepas patógenas pueden transferir su poder patogénico a otras cepas normalmente inofensivas. De aquî que incluso la tipificación de los bacilos Coli encontradas en la deposición no nos va a dar seguridad sobre su poder patógeno $(25,29)$, lo que podría hacer aconsejable el tratamiento antibiótico generalizado.

En contra de esta posición hay, sin embargo, argumentos poderosos:

1) La mayoría de las diarreas agudas, en una proporción superior al $60 \%$, no son bacterianas.

2) En un porcentaje importante, las diarreas agudas son acompañadas de otras infecciones extraintestinales, que pueden ser enmarcadas por el uso precoz de antibióticos absorbibles. Los antibióticos no absorbibles serían pocos eficaces en las infecciones entéricas por gérmenes con capacidad invasora de mucosa (32).

3) Los antibióticos alteran la flora intestinal normal, que protcge contra la invasión por gérmenes patógenos, en espccial Salmonellas.

4) El uso de antibióticos crea cepas resistentes a ellos, ya sea por mutación y selección natural o por transferencia de episomas entre cepas (33).

5) El tratamiento antibiótico parece ser inefectivo contra las diarrcas por Salmonellas $(25,34)$.

Por todas estas razones, pensamos que sólo deben tratarse con antibióticos al ingreso los lactantes con deposiciones de tipo enterocólico.

El tratamiento debiera intaurarse también si la hidratación no se consigue en el plazo de 48 horas y/o si las deposiciones persisten líquidas más allá de las 72 horas. En cstos casos, es previa la toma de muestras para cultivos de deposiciones, orina, sangre y médula ósea, así como también un frotis de deposiciones para investigación de polinucleares (25) y cualquier otro examen de laboratorio que se considere pertincnte.

Infecciones cxira intestinales: Se confirmaron o sopecharon en el 44\% de los lactantes con evolución tórpida de su diarrea, sien- 
do las más frecuentes la septicemia y la infección urinaria. Frente a estos enfermos, se nos ha planteado la duda si ellos deben mantenerse en el grupo diagnóstico de diarreas agudas, o si este síndrome constituye en ellos sólo una manifestación más de otra patología.

En este segundo caso la diarrea debcría considerarse parenteral, según el concepto introducido por Czerny. Aunque la patogenia de este cuadro no ha sido nunca bien aclarado, $\mathrm{y}$ persisten diversas teorías al respecto (36), es indudable que se trata de una realidad en muchos casos. En las escasas publicaciones sobre septicemias a Gram (-) cn nuestro medio $(37,38)$, la diarrea aguda con o sin deshidratación aparece como uno de los síntomas más constantes. Es conocido que una de las formas clínicas de presentación de las infecciones urinarias y de las meningitis purulenta es como una diarrea aguda con deshidratación.

Por otra parte, es posible que una infección bacteriana intestinal constituya el punto de partida para el paso de ellos a la sangre y su localización secundaria en otros órganos. Como ejemplo, en Israel (40) se encontró Coli en los pulmones de lactantes fallecidos por diarrea, utilizando la técnica de inmunofluorecencia.

Es importante recordar, en todo caso, que en las diarreas agudas con deshidratación de evolución tórpida deben agotarse los esfuerzos para pesquisar una septicemia u otro foco de infección.

Intolerancia a la lactosa. El problema de las alteraciones de las disacaridasas intestinales secundarias a diarreas agudas ha sido estudiado en los últimos años por numerosos autores extranjeros y nacionales $(40,41,42$, $43,44,45)$. En nuestro análisis se comprobó o sospechó su existencia en el 9,7\% de los lactantes con diarreas de evolución tórpida. Aunque esta proposición es semejante al $11 \%$ encontrado por Soriano (15), parece reducida de acuerdo a la experiencia de otros autores. Ello podría explicarse por varias razones.

a) Nuestro programa era estudiar la intolerancia a hidratos de carbono en todos estos niños, de acuerdo al esquema sugerido por Soriano $(15,45)$ y Guiraldes (46). Sin embargo, ello fue posible sólo en un número reducido de enfermos.

b) Como test de orientación usamos un $\mathrm{pH}$ en deposiciones de 5 ó menos, de acuerdo con Guiraldes (46), en tanto que otros autores consideran significativo un $\mathrm{pH}$ de 6 o menos $(15,47)$.

c) Una parte importante de los lactantes hospitalizados en nuestro servicio por diarrea aguda con deshidratación, fueron realimentados con una mezcla de zanahoria, quesillo, aceite y azúcar, muy pobre en lactosa. La intolerancia a esta azúcar no pudo evidenciarse por esto en esos niños.

Es posible, que la alteración secundaria de las disacaridasas intestinales haya sido más frecuente que lo que demuestran las cifras obtenidas. Sin embargo, pensamos que aún así su importancia como causa de evolución tórpida de las diarreas, es inferior a la infección.

\section{RESUMEN Y CONCLUSIONES}

1. Se revisan las fichas clínicas de todos los niños ingresados a la Unidad de lactantes del Hospital Sótero del Río entre el $1^{\circ}$ de octubre de 1974 y el 28 de febrero de 1975, encontrándose 253 en que el motivo de la hospitalización fue de una diarrea aguda con deshidratación.

2. El 70\% de estos niños eran menores de 6 meses, el $60 \%$ desnutridos grado II y III $y$ el $60 \%$ presentaba una deshidratación con natremia normal, en tanto que habia hipernatremia en el 4,7\% e hiponatremia en el $16,9 \%$ de ellos.

3. La letalidad en el grupo estudiado fue de $5,5 \%$ que se compara con otras experiencias nacionales.

4. El estado de shock al ingreso aumenta el riesgo de morir en 5 veces. También aumenta este riesgo en los desnutridos grado III, aunque no en forma estadísticamente significativa.

5. La evolución del cuadro diarreico fue tórpida, según criterio que se define, en el $36,5 \%$ de los niños, apareciendo esta modalidad evolutiva influenciada sólo por el carácter disenteriforme de la diarrea y no por otras características analizadas.

6. La investigación de las causas de evolución tórpida reveló que pueden clasificarse en 4 grandes grupos:

a) infecciones bacterianas enterales enteropatógenas en el $45 \%$ de los casos. 
b) infecciones extraintestinales en el 44\% de ellos, equivalente septicemias e infecciones urinarias.

c) recaidas intrahospitalarias en el 12\%.

d) intolerancia secundaria a lactosa en el $10 \%$.

7. De acuerdo a estos resultados, se discute la conveniencia de iniciar tratamiento con antibióticos de rutina en todos los lactantes ingresados con este diagnóstico.

8. Se discute también si en los casos con infecciones extraintestinales, la diarrea debe considerarse como causa o como síntoma.

9. Finalmente, se revisan las posibles explicaciones para el bajo número de lactantes con diarrea aguda de evolución tórpida en que ésto se atribuyó a intolerancia secundaria a lactosa.

\section{R E F E R E N C I A S}

1.-Puentes, R.: "Mortalidad Infantil en Chile. Avances y 'Problemas actuales". Rev. Chilena Pediatría, vol. 44 № 2: 169-176, 1973.

2.-Meneghello, J. y Macaya, J.: Tendencia de la mortalidad por diarrea en el lactante en los últimos 20 años. Pediatría 15: 465, 1972.

3.- Servicio Nacional de Salud. Subdepartamento de Fomento de la Salud y Estadística. 1974.

4.- Servicio Nacional de Salud. Anuario "Egresos hospitalarios", 1968.

5.- Servicio Nacional de Salud. Area Sur Oriente. Oficina de Estadística.

6.-Macaya, J.; Duffau, G. y Soriano, H.: Sindrome diarreico agudo con deshidratación del lactante. Evolución tórpida. Pediatría 15: 488, 1972.

7.-Scroggie, A.: Clínica, prevención y tratamiento del sindrome tóxico del lactante. Rev. Chilena Pediatría 46: 274, 1955.

8.-Legarreta, J.: Algunos aspectos de los trastornos nutritivos agudos en el niño. Rev. Chilena Pediatría 20: 147, 1949.

9.-Meneghello, J.; Aguiló, C.; Monckeberg, F.; Ceruti, E.; Soriano, H.; Rubio, S. y Fernández, $E$.: Análisis de 244 lactantes con deshidratación aguda grave. Pediatría 5: 431, 1962.
10.-Jarpa, S.; Martner, J.; Cabrejo, C.; Córdova, J. M. y Segura, Z. E.: Influencia de algunos factores etiopatogénicos en la evolución y letalidad de la diarrea aguda del lactante. Rev. Ch. Ped. 41: 324, 1970.

11.-Puostilo, DI an Cannon, DH: "Fatal infections in protein-caloric malnurished children with thymolymphatic atrophy". Arch. of Diseases in Childhood, 50: 149, 1975.

12.-Mac Fortane, H.: "Cell-mediated inmunity in P-C malnutrition". Lancet, 2: 1146, 1971.

13.-Gordon, J. E. and Scrimshaw, N. S.: Infections disease in the malnurished. Med. Clin. N. Amer. 54 : 1495, 1970.

14.-Avery, G. B.; Villavicencio, O.; Lilly, J. R. and Randolph, J. G.: Intractable diarrhea in early infancy. Ped. 41: 712, 1968.

15.-Soriano, H.; Macaya, J.; Duffau, G.; García, I. y Urbina, A. M.: Sindrome diarreico agudo del lactante. Pediatría 15: 452, 1972.

16.-Wu, E.; Contreras, G.; Ohlbaum, A.; Gallo, A.; González, C. y Gilabert, B.: Estudios sobre la etiología viral de las diarreas agudas del lactante. Rev. Ch. Ped. 42: 221, 1971.

17.-Montoya, C.; Gilabert, B.; Simón, C. y Sana, A.: Etiología de las diarreas infantiles. Rev. Chilena Pediatría 35: 923, 1964.

18.-Lobos, H.; Aguilar, C. y Romero, H.: Bacteriología de las diarreas agudas en el lactante hospitalizado. Pediatría 8: 280, 1965.

19.-Cramblett, H. G. and Sievers, C. M. F.: The etiology of gastroenteritis in infants and children. Ped. 35: 885, 1965.

20.-Ramos Alvarez, M. and Sabin, A.: Enteropathogenia viruses and bacteria in diarrhea. J.A.M.A. 167 № 2, suppl. 10, 1958.

21.-Ramos Alvarez, $M$. and Olarte, J.: Diarrheal diseases of children. Am. J. Dis. Child. 107: 218, 1964.

22.-Prenzel, Y. y Duarte, E.: Bacteriología de las diarreas. Hospital Roberto del Río 1969-71. Pediatría 16: 92, 1973.

23.-Prado, V.; Mimica, I.; Eduardo, M.: "Etiología Bacteriana de la diarrea aguda del lactante. Aportes de la Inmunofluorescencia. Estudios 
de sensibilidad in vitro de los bacterios patógenos aislados". Rev. Chilena Pediatría. Vol. 45: 135-142. 1974.

24.-Prado, V.; Mimica, I.: "Estudio comparativo doble ciego de los efectos terapéuticos de neomicina, lactobacilo y placebo en infección enteral por Escherichia Coli Enteropatógeno". Rev. Chilena Ped. vol. 45, 2: 143-146, 1974.

25.-Errázuriz, O.; Gilardino, J. y Pino, M.: Diarrea aguda del lactante. Rev. Ch. Ped. 45: 209, 1974.

26.-Drackman, R. H.: Acute infectious gastroenteritis. Ped. Clin. of North Am. 21, N: 5: 711, 1974.

27.-Schrciber, D. S.; Blacklow, N. R. and Trier, J. $S .:$ The mucosal lesion of the proximal small intestine in acute infectious nombacterial gastroenteritis. The New England. J. Mcd. 238: 1318, 1973.

28.-Smith, H. W. and Halls, Sh.: Studies on E. Coli anterotoxin. J. Path. Bact. 93: 531, 1967.

29.-Takauchi, A. and Spring, H.: Electron microscope studies of experimental infection in the preconditioned guinea pig. Am. J. Path. 51: 137, 1967.

30.-Dum Pont, H. L.; Hornick, R. S. ct al.: Pathogenesis of E. Coli diarrhea. New Eng. J. Med. 285: 1, 1971.

31.-Soriano, H. y Macaya, J.: Sndrome Diarreico Agudo. En Texto de Pediatría, J. Mencghello. Intermédica, Buenos Aires, 1972.

32.-Watkins, J.: Bila acid metabolism and fat absortion in newborn infants. Ped. Clin. North. Am. $21 \mathrm{~N}$ 2: 501, 1974.

33.-Haltaton, K. C.; Nelson, J. D.; Hinton, L. V. et al. Comparison of orally absorbabie and nonabsorbable antibiotics in shigellosis. J. of Ped. 72: 708, 1968.

34.-Gardner, P.: Antimicrobial drug theray in pediatric practice. Ped. Clin. of North Am. 21, N 3: 617, 1974.

35.-Weissman, J. et at. Shigellosis, To treat or not treat. JAMA. 229: 1215, 1974.

36.-Clyde, W. A.: Salmonellosis in infants and children. Ped. 19: 175, 1957.
37,-Royer, P.; Mass, N. P. et Pringuet, C.: Les diarrhés microbiennes du nourrison. Centre International de L'enfance. 'Paris 1960.

38.-Del Río, L.; Caballero, C.; Eglinton, G. y Cassorla, E.: Septicemias por bacilos Gram- negativos en el lactante. Rev. Ch. Ped., 37: 343, 1966.

39.-Olcese, A. y Dal'Borgo, P.: Sepsis por bacilos gram negativos en el lactante. Rev. Ch. Ped. 42, 605, 1971.

40.-Moshe, M., Drucker et al.: Inmunofluorescent demonstration of enteropathogenie. E. Coli in tissues of infants duyin with enteritis. Ped. $46: 855,70$.

41.-Dahlgvist, A.: General Surrey on the digestion and absortion of carbohidrates". Simposia of the Swcdish Nutrition Foundation. XI.9-14, 1973.

42.-Crane, R.: "Digestive-Absorptive organization in the brush border membrane". Simposia of the Swedish Nutrition Foundation XI-15-20. 1973.

43.- Rosenzweig, N.: The influence of dietary carbohidrats on intestinal disaccharidase activity in man. Simposia of the Suvedish Nutrition Foundation XI 21, 31, 1973.

44.-Cundmand-Hoger, E.: "Clinical significance and some nutritional aspects of disacaharide malabsorption". Simposia of the Swesian Nutrition Foundation XI. 37, 44, 1973.

45.-Soriano, H.; Macaya, J.; Duffau, G.; Garcia, Y. y Urbina, A. N.: Empleo de la reacción de Fehling en el diagnóstico de la intolerancia a los disacáridos. Pediatría 15: 452, 1972.

46.- Guiraldes, E.: Comparación entre diversos métodos para diagnóstico rápido de malabsorción de hidratos de carbono. Rev. Ch. Ped. 45: 399, 1974.

47.-Emilfork, M. y Duffau, G.: Intolerancia secundaria a hidratos de carbono. Metódica de estudio. Pediatría 17: 1974.

48.-Calderón, A.; Goldenberg, E.; Macaya, J. y cols.: Sindrome diarreico agudo de evolución tórpida. Análisis clínico de 100 pacientes. Pediatría 17: 95, 1974. 